\title{
Origine et impact du chenal Tchad-Cameroun sur la propagation de Chromolaena odorata (L.) R. M. King and H. Robinson (Asteraceae) de Meiganga à Touboro
}

\author{
B. NGOTTA BIYON ${ }^{1}$, M. TCHATAT ${ }^{2}$, E.S. MOKAKE ${ }^{1}$ et D.S. DIBONG ${ }^{1,3,4 *}$ \\ ${ }^{1}$ Département de Biologie des Organismes Végétaux, Faculté des Sciences, B.P. 24157, Université de \\ Douala, Cameroun. \\ ${ }^{2}$ Institut de la Recherche Agricole (IRAD), B.P. 2123, Yaoundé, Cameroun. \\ ${ }^{3}$ Institut des Sciences Halieutiques, B.P. 2701, Université de Douala, Cameroun. \\ ${ }^{4}$ Département des Sciences Pharmaceutiques, Faculté de Médecine et des Sciences Pharmaceutiques, \\ B.P. 2701, Université de Douala, Cameroun. \\ *Auteur correspondant ; E-mail : didierdibong@yahoo.fr
}

\section{RESUME}

L'objectif de ce travail est de déterminer l'origine et les effets du chenal Tchad-Cameroun sur la propagation de Chromolaena odorata, Asteraceae, considérée comme mauvaise herbe dans la zone d'étude. La méthodologie suivie a comporté trois étapes: la prospection générale de la zone d'étude, le choix des itinéraires d'étude et la confection des relevés floristiques de Chromolaena odorata. L'espèce héliophile est présente dans toute la zone d'étude. Dans l'itinéraire 1, de Garoua Boulaï à Meiganga (Centrafrique vers Cameroun), l'abondance-dominance et le recouvrement moyen ont des maxima. Les indices d'abondancedominance et de recouvrement moyen élevés hors chenal dans cet itinéraire justifient l'origine centrafricaine de cette espèce, d'où le nom commun de «bokassa » utilisée par les populations pour la désigner. De Meiganga à Mbaï-mboum (itinéraire 2), Chromolaena odorata recouvre également plus de 3/4 de la surface et les individus occupent 87,5\% de cet itinéraire. De Mbaï-mboum à Ngaoundéré (itinéraire 3), les deux indices décroissent simultanément montrant l'influence du chenal sur la résurgence et l'importance de Chromolaena odorata. Les méthodes culturales utilisées pour éradiquer $C$. odorata sont la coupe des tiges de la plante associée à un dessouchage des organes souterrains.

(C) 2014 International Formulae Group. All rights reserved.

Mots clés : Chromolaena odorata, origine, impact, chenal, propagation.

\section{INTRODUCTION}

Chromolaena odorata (L.) R. M. King and H. Robinson (Asteraceae) est une plante herbacée pérenne formant des buissons denses et enchevêtrés de 1,5-2 $\mathrm{m}$ de hauteur et atteignant $6 \mathrm{~m}$ lorsque le port est arborescent (Ngobo et al., 2004 ; Koutika et Rainey,
2010). L'espèce est originaire de l'Amérique tropicale et subtropicale et a une large distribution du sud des Etats-Unis au nord de l'Argentine (Ngobo et al., 2004). Introduite dans les années 1940 comme plante de couverture en plantation de cacao, la plante est devenue envahissante en Afrique tropicale, 
au Ghana, au Nigéria, au Cameroun, en Centrafrique, en Côte d'Ivoire, mais également en Afrique du Sud (Timbilla et Braimah, 2000; Molard, 2004). Depuis plusieurs décennies, la présence de Chromolaena odorata pose d'énormes problèmes dans les pays d'Asie du Sud-Est et d'Afrique sub-saharienne en général, et au Cameroun en particulier. Les travaux menés au sud du Cameroun, plus précisément dans la région de l'Est, indiquent que Chromolaena odorata est apparue à Kandara $\left(4^{\circ} 20 \mathrm{~N}\right.$ et $13^{\circ} 46$ E) en 1969 à la faveur de la création de la piste, mais n'a commencé à être envahissante qu'en 1973. Les travaux menés en Centrafrique par Boutrais en 1988 montrent que l'incidence néfaste de Chromolaena odorata sur les terroirs pastoraux a été observée à Baboua dans les années 1980. Cette plante est responsable de l'empoisonnement du bétail dans les terroirs pastoraux (Achoundong et al., 2000).

Baboua est une ville frontalière $\mathrm{du}$ Cameroun, voisine de Garoua-Boulaï. Ces deux localités présentent des altitudes similaires et par leur proximité, la présence de Chromolaena odorata à Baboua suggère sa présence à Garoua-Boulaï à la même époque. La région de l'Adamaoua est une zone importante d'élevage. En 2004, le cheptel comptait environ 5,9 millions de bovins, 3,8 millions d'ovins et 4,4 millions de caprins. L'élevage diminue ces dernières années à cause de la présence de Chromolaena odorata dans les pâturages de cette région. Cette espèce colonise les savanes en s'élevant plus haut que les Graminées qui les constituent (Ngotta Biyon, 2010). Les graines de Chromolaena odorata pouvant rester enfouies dans le sol pendant une durée de 5 ans
(Vanderwoude et al., 2005), on s'attend à ce que l'on ait une forte réapparition de cette Asteraceae dans les endroits ayant subi un retournement du sol par les engins lourds, notamment sur le chenal pétrolier TchadCameroun. Au cours des travaux de construction du pipeline Tchad-Cameroun, les entreprises retenues avaient débuté la pose du pipeline dans le sud en allant vers le point triple (région de Touboro).

L'objectif du présent travail est: (1) de déterminer l'origine de Chromolaena odorata; (2) de recenser les facteurs de dissémination et (3) d'évaluer l'impact du chenal sur la propagation de cette Astéracée.

\section{MATERIEL ET METHODES}

Site d'étude

Le secteur d'étude est à cheval entre les régions de l'Adamaoua et du Nord. Les coordonnées géographiques sont comprises entre $06^{\circ} 31^{\prime} 00$ et $07^{\circ} 46^{\prime} 60 \mathrm{~N}, 1^{\circ} 18^{\prime} 00$ et $15^{\circ} 2200$ E. L'altitude moyenne est de $988 \mathrm{~m}$. Deux types de climat sont observés:

- dans la zone de Meiganga-Ngaoundéré, le climat est de type tropical d'altitude à deux saisons, caractérisé par des précipitations encore abondantes ; c'est un régime tropical humide avec une saison sèche d'au moins 4 mois ;

- dans la zone de Touboro, le climat est de type soudano-guinéen caractérisé par une saison sèche qui dure environ 6 mois.

De même, deux types de savane sont distingués, les savanes périforestières dans le pourtour de Meiganga et les savanes arborées ou arbustives soudano-guinéennes au nord de cette ville:

- les savanes périforestières font le passage de la forêt à Sterculiaceaes aux savanes de 
1'Adamaoua. Elles comprennent les savanes de la zone post forestière congo-guinéenne et les savanes soudano-guinéennes de transition ; - les savanes arborées ou arbustives à Daniellia oliveri avec un tapis graminéen d'Andropogonées, très sensibles à l'action de l'Homme. Certains arbustes ou petits arbres sont localement très abondants et créent des faciès particuliers. Des vallons forestiers, actuellement intensément défrichés, possèdent encore une flore rappelant la forêt semicaducifoliée à Sterculiaceaes (Ngotta Biyon, 2010).

\section{Méthodes}

Trois étapes ont été suivies sur le terrain :

- la prospection générale de la zone d'étude ;

- le choix des itinéraires d'étude ;

- la confection des relevés floristiques de Chromolaena odorata.

\section{Prospection de la zone d'étude}

Les prospections ont été conduites pendant deux semaines, par voie routière et à pied de Meiganga à Ngaoundéré en passant par Touboro et Tcholliré, localités limitrophes de la zone d'étude. La superficie couverte a été de $21600 \mathrm{~km}^{2}$ et la distance parcourue de $577 \mathrm{~km}$ (Figure 1).

\section{Confection des itinéraires d'étude et des relevés floristiques}

Trois itinéraires possédant quatre stations chacun ont été confectionnés à partir du tracé approximatif du pipeline Tchad-
Cameroun (Figure 2). Dans chaque station, un relevé a été effectué dans une surface de 100 $\mathrm{m}^{2}$ en utilisant trois indices parmi ceux définis par Braun-Blanquet (1932) pour caractériser la propagation de Chromolaena odorata: la présence, l'abondance-dominance et le recouvrement moyen. L'indice d'abondancedominance permet de déterminer le degré de recouvrement d'une espèce dans un relevé ou dans un site comportant plusieurs relevés. Pour une aisance pratique, Braun-Blanquet (1932) a introduit le coefficient mixte d'abondance-dominance allant de + à 5 :

+ : individus rares couvrant moins de $1 / 20^{\mathrm{e}}$ de la surface ;

1: individus moins nombreux occupant $1 / 20^{\mathrm{e}}$ de la surface ;

2 : individus abondants couvrant de $1 / 20^{\mathrm{e}}$ à $1 / 4$ de la surface ;

3 : individus recouvrant de $1 / 4$ à $1 / 2$ de la surface ;

4 : individus recouvrant $1 / 2$ à $3 / 4$ de la surface ;

5 : individus recouvrant plus de $3 / 4$ de la surface.

Ces indices sont ensuite traduits en recouvrement moyen (RM) :

+ : individus occupant $0,5 \%$ du territoire ;

1 : individus occupant $3 \%$ du territoire ;

2 : individus occupant $15 \%$ du territoire ;

3 : individus occupant $37,5 \%$ du territoire ;

4 : individus occupant $62,5 \%$ du territoire ;

5 : individus occupant $87,5 \%$ du territoire.

La présence est notée + et l'absence -. 


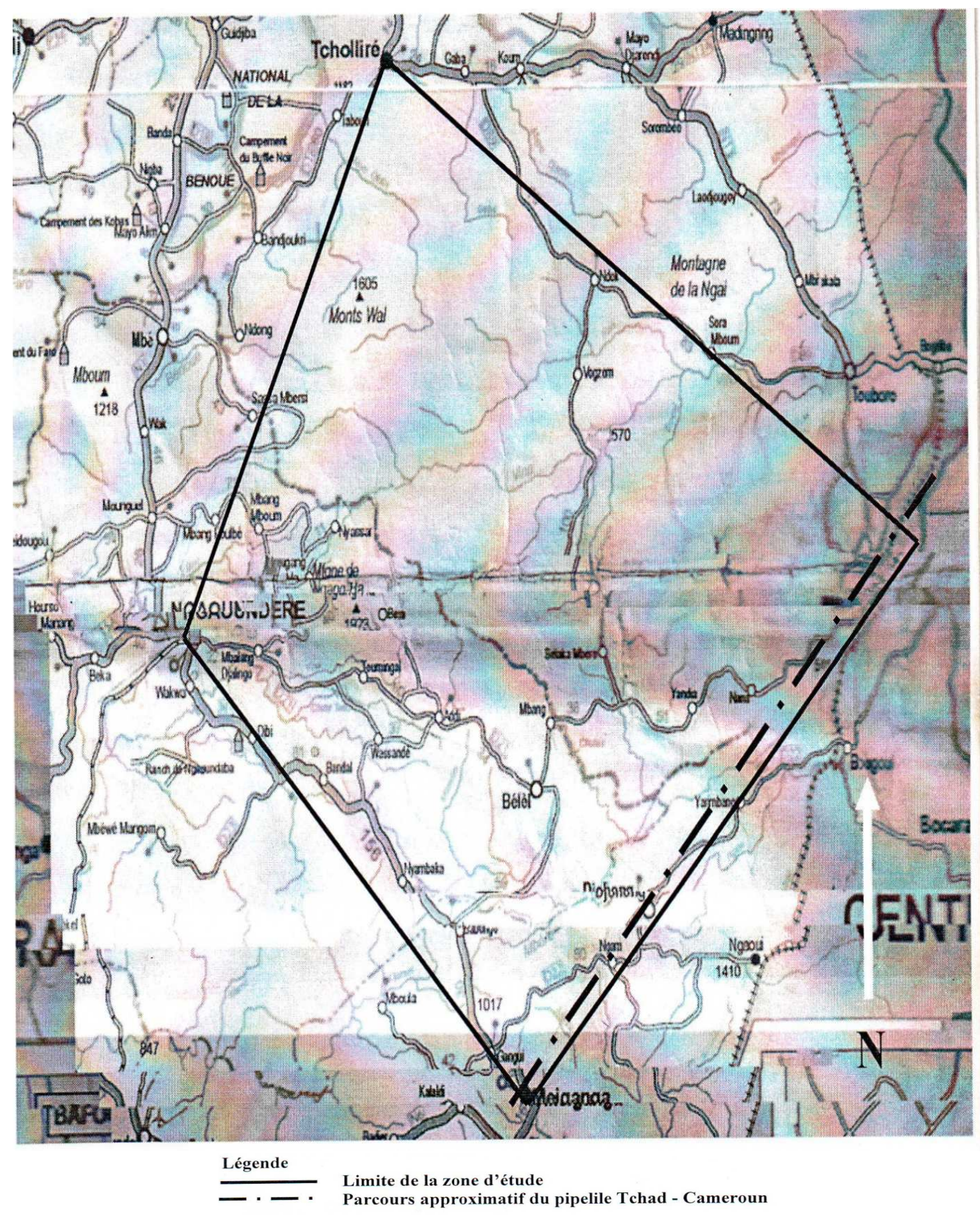

Figure 1: Extrait de la carte routière du Cameroun au 1/1 500000. 


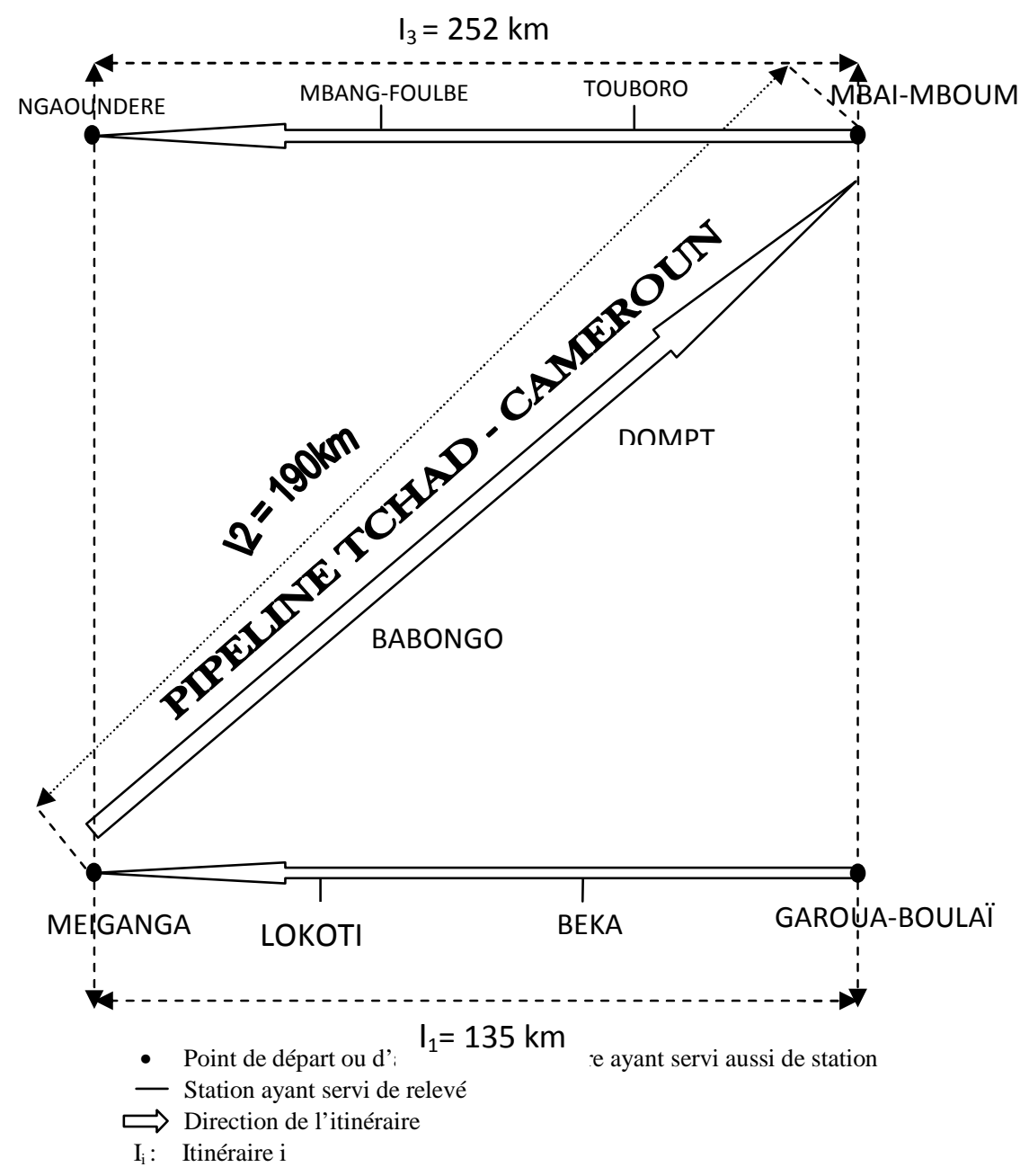

Figure 2 : Itinéraires parcourus sur le terrain.

\section{RESULTATS}

Chromolaena odorata est présente dans toute la zone d'étude. Dans l'itinéraire 1, de Garoua Boulaï à Meiganga (Centrafrique vers Cameroun), l'abondance-dominance et le recouvrement moyen ont des maxima (Tableau 1). La plante a un potentiel d'envahissement suffisamment important pour conquérir les savanes peu perturbées de Garoua Boulaï jusqu'aux espaces ouverts par le passage du chenal. L'espèce, héliophile se développe et produit des fourrés denses monospécifiques de la frontière centrafricaine (Garoua Boulaï) au chenal (Meiganga) où elle est soumise à un ensoleillement direct. Les indices d'abondance-dominance et de recouvrement moyen élevés hors chenal dans cet itinéraire justifient l'origine centrafricaine de cette espèce, d'où le nom commun de «bokassa » utilisée par les populations pour la désigner. Ce développement important limite les espaces pour la régénération des plantes indigènes et par conséquent induit une perturbation des cycles de développement et 
de succession des espèces locales. Le développement de $C$. odorata a pour conséquence la perturbation du cycle naturel de régénération et de succession végétale des graminées, plantes fourragères très appréciées par le bétail dans la région. De plus, dans l'environnement immédiat du chenal, le développement de $C$. odorata produit une strate monospécifique inexistante avant son invasion.

De Meiganga à Mbaï-mboum (itinéraire 2: aval vers l'amont du chenal), Chromolaena odorata recouvre également plus de $3 / 4$ de la surface et les individus occupent $87,5 \%$ de cet itinéraire (Tableau 2).

De Mbaï-mboum à Ngaoundéré (itinéraire 3 : chenal vers l'intérieur du pays), les deux indices décroissent simultanément montrant l'influence du chenal sur la résurgence et l'importance de Chromolaena odorata (Tableau 3). La perturbation et la modification de l'équilibre écosystémique par le chenal ont provoqué l'abandon des pâturages naturels par les populations concernées.

Les méthodes de culturales utilisées pour éradiquer $C$. odorata sont la coupe des tiges de la plante associée à un dessouchage des organes souterrains. La simple opération de coupe n'est pas suffisante car le système racinaire contient suffisamment d'énergie pour produire les rejets.

Tableau 1 : Relevés floristiques de Chromolaena odorata le long de l'itinéraire 1, de Meiganga à Garoua-Boulaï.

\begin{tabular}{lcccc}
\hline Localités & Garoua-Boulaï & Beka & Lokoti & Meiganga \\
\hline Distance $(\mathrm{km})$ & 0 & 75 & 103 & 135 \\
\hline Présence & 1 & 1 & 1 & 1 \\
\hline $\begin{array}{l}\text { Abondance- } \\
\text { Dominance }\end{array}$ & 4 & 4 & 4 & 5 \\
\hline $\begin{array}{l}\text { Recouvrement } \\
\text { Moyen }(\%)\end{array}$ & 62,5 & 62,5 & 62,5 & 87,5 \\
\hline
\end{tabular}

Tableau 2 : Relevés floristiques de Chromolaena odorata le long de l'itinéraire 2, de Meiganga à Mbaï-mboum.

\begin{tabular}{lcccc}
\hline Localités & Meiganga & Babongo & Dompta & Mbaï-mboum \\
\hline Distance $(\mathrm{km})$ & 135 & 175 & 275 & 325 \\
\hline Présence & 1 & 1 & 1 & 1 \\
\hline $\begin{array}{l}\text { Abondance- } \\
\text { Dominance }\end{array}$ & 5 & 5 & 4 & 4 \\
\hline $\begin{array}{l}\text { Recouvrement } \\
\text { Moyen }(\%)\end{array}$ & 87,5 & 62,5 & 62,5 & 62,5 \\
\hline
\end{tabular}


Tableau 3 : Relevés floristiques de Chromolaena odorata le long de l'itinéraire 3, de Mbaï-mboum à Ngaoundéré.

\begin{tabular}{lcccc}
\hline Localités & Mbaï-mboum & Touboro & Mbang-Foulbé & Ngaoundéré \\
\hline Distance $(\mathrm{km})$ & 325 & 275 & 93 & 73 \\
\hline Présence & 1 & 1 & 1 & 1 \\
\hline $\begin{array}{l}\text { Abondance- } \\
\text { Dominance }\end{array}$ & 4 & 4 & 3 & 3 \\
\hline $\begin{array}{l}\text { Recouvrement } \\
\text { Moyen (\%) }\end{array}$ & 62,5 & 62,5 & 37,5 & 37,5 \\
\hline
\end{tabular}

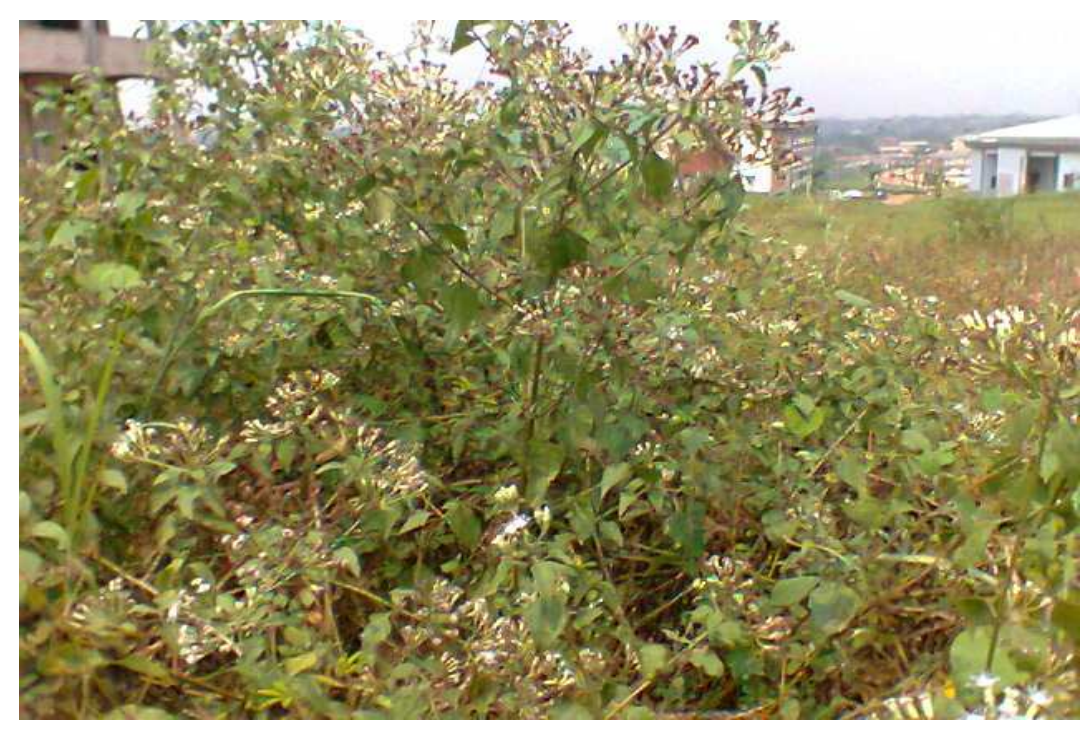

Figure 3: Appareil végétatif de Chromolaena odorata présentant des fleurs et des fruits. (Photo Ngotta Biyon, 2014).

\section{DISCUSSION}

Dans les zones de prédilection, Chromolaena odorata est considérée comme une mauvaise herbe notamment dans la zone d'étude (Ngobo et al., 2004 ; Koutika et Rainey, 2010). De Meiganga à Touboro, $C$. odorata colonise les pâturages très rapidement. Par ailleurs, l'espèce renferme dans ses feuilles et tiges des huiles volatiles qui lors d'une longue période de saison sèche peuvent augmenter de façon importante le régime des risques d'incendies (Koutika, 2010). Certaines surfaces de pâturages naturels ont été abandonnées le long du chenal à la faveur de l'invasion et des villages entiers ont été parfois abandonnés (Aterrado et Bachiller, 2002). Elle est également signalée dans les pâturages au Nigéria, aux Philippines, en République de Centrafrique et à Java (Ngotta Biyon, 2010). Aux Philippines où elle a été introduite récemment, $C$. odorata colonise les pâturages naturels très rapidement. Selon les éleveurs, des cas d'empoisonnement du bétail ont été observés à certains moments de l'année.

Plusieurs facteurs biotiques et abiotiques sont favorables à la propagation de cette mauvaise herbe dont le cycle biologique, 
la survie dans les conditions défavorables, la capacité d'adaptation et les invasions involontaires.

Les fleurs de $C$. odorata sont hermaphrodites. La floraison est saisonnière et se produit en saison sèche (Zachariades et al., 2009). Les fleurs sont pollinisées par de nombreux insectes non spécialisés. La production des graines est très importante et de fortes densités peuvent être atteintes (supérieure à 2000 graines $/ \mathrm{m}^{2}$ ). Une importante compétition intraspécifique se déroule lors des premiers mois de développement (Ngotta Biyon, 2010). La maturité des graines arrive à son terme en février-mars. La durée de production est d'environ deux mois et correspond à la période de maturation de l'ensemble des graines de la plante (Molard, 2004). La dormance et l'ombre sont nécessaires pour la germination justifiant la résurgence de la plante après retournement du sol par les engins. Il est possible que les branches tombées au sol développent des racines adventives adaptées à la reproduction végétative (Ngotta Biyon, 2010). C. odorata a un pouvoir de régénération important. De nombreux rejets de souches ont été observés après perturbation de la plante par les coupes (Molard, 2004).

La dispersion des fruits de $C$. odorata est principalement anémochore bien qu'elle soit aussi exozoochore et anthropochore. Les fruits se collent facilement aux fourrures, plumages ou habits, véhicules et matériel agricole. Ces deux types de dispersion assurent une propagation efficace des graines de $C$. odorata sur de courtes et longues distances (Molard, 2004). La dissémination de la plante est rapide et elle peut coloniser en deux ans un milieu nu à partir des foyers restreints composés de quelques individus (Molard, 2004).

La grande quantité de graines produites et la variabilité de la dormance contribuent à la survie durant les périodes difficiles (feu, désherbages efficaces, fermeture du couvert). C. odorata a un spectre de tolérance pédologique très important. Elle peut se développer sur des sols allant des dunes de sables aux terrains lourds argileux (Timbilla et Braimah, 2000). Cependant, elle semble préférer les milieux bien drainés. Les exigences pluviométriques annuelles de $C$. odorata sont celles de la zone d'étude (1000 et $2500 \mathrm{~mm}$ ). La plante supporte une période sèche marquée de 4 à 5 mois (Molard, 2004).

Le déplacement des véhicules dans les zones colonisées où des graines sont présentes au sol a favorisé la dissémination de la plante (Ngotta Biyon, 2010). Le rôle joué par le déplacement des troupeaux et les engins de déblayage qui ont retourné le sol, le long chenal (tracé du pipeline) a été déterminant. La plante peut également avoir été disséminée par les déplacements fréquents et incessants des personnes construisant le pipeline.

Les opérations culturales doivent être réalisées au moins deux fois durant la saison de pousse (Timbilla et Braimah, 2000). L'utilisation du feu est à proscrire car elle favorise les rejets de la plante et lui donne un avantage compétitif grâce à ses organes souterrains vigoureux. La lutte biologique peut être expérimentée avec succès car la plante colonise aussi bien les milieux cultivés que les aires naturelles. Au Ghana, l'introduction du contrôleur a permis la réduction de l'occupation du sol par $C$. odorata de 85 à $35 \%$ ainsi qu'un développement de la biodiversité (Timbilla and Braimah, 2000). De même, la lutte chimique adaptée pour les milieux cultivés, mais difficilement réalisable dans les zones naturelles peut être expérimentée avec succès (Molard, 2004). Cependant, une lutte efficace contre cette plante consiste à agir plus tôt selon une démarche d'éradication précoce.

\section{Conclusion}

Chromolaena odorata est présente en grande quantité en savane arbustive (région de 
Meiganga), mais dans la savane arborée (Touboro), cette plante se limite à quelques pieds isolés ou à quelques individus groupés. Les facteurs à la fois biotiques et abiotiques favorisent la dissémination de l'Astéracée dans la zone d'étude. Le chenal pétrolier Tchad-Cameroun n'est pas à l'origine de la propagation de Chromolaena odorata dans la zone étudiée; néanmoins il a facilité la résurgence de cette plante.

\section{REMERCIEMENTS}

Notre profonde gratitude à la $\mathrm{COTCO}$ Cameroun qui a financé cette recherche.

\section{REFERENCES}

Achoundong G, YoutaHappi J, Bonvallot J, Guillet B. 2000 - Formation et évolution des recrûs en savane. In Dynamique des Ecosystèmes Forestiers Intertropicaux. Ed Orstom-CNRS : Paris; 115 - 119.

Aterrado ED, Nemesis SD, Bachiller. 2000. A classical approach to biological control of the weed Chromolaena odorata King \& Robinson (Asteraceae). 3 p.

Braun-Blanquet J. 1932. Plant Sociology. The Study of the Plant Communities. McGraw-Hill: New-York; 405 p.

Koutika LS, Rainey HJ. 2010. Chromolaena odorata in different ecosystem: weed or fallow plant? Applied Ecology and Environmental Research, 8(2): 131-142.

Molard G. 2004. Analyse du Risque Phytosanitaire. Programme Poseidon, CIRAD, 45 p.

Ngobo M, Mc Donald M, Weise S. 2004. Impacts of type of fallow and invasion by
Chromolaena odorata on weed communities in crop fields in Cameroon. Ecology and Society 9(2): 1. (online): http//www.ecologyandsociety.org/vol19/a rt1

Ngotta Biyon B. 2010. Origine et propagation de Chromolaena odorata (L.) R. M. King and H. Robinson (Asteraceae) entre Meiganga et Mbaï-mboum dans le nord Cameroun. Mémoire de Maîtrise de l'Université de Douala, 50p.

Timbilla JA, Braimah H. 2000. Establishment spread and impact of Pareucaetes pseudoinsulata (Lepidoptera: Arctiidae) an exotic predator of the siam weed, Chromolaeana odorata (Asteraceae: Eupatoriae) in Ghana, Proceedings of the $\mathrm{X}$ International Symposium on Biological Control of Weeds 4-14 July 1999, Montana State University, Bozeman, Montana, USA. Neal R. Spencer Ed., pp 105-111.

Vanderwoude C, Scalan JC, Davis B, Funkhouser S. 2005. Plant for national delimiting survey for siam weed. Natural Resources and Mines Land Protection Services, Queensland Government.

Zachariades CM, Day R, Muniappanet GVP Reddy. 2009. Chromolaena odorata (L) King and Robinson (Asteraceae).In Biological Control of Tropical Weeds using Anthropod. Muniappan R, Reddy GVP, Raman A (eds). Cambridge University Press: Cambridge, UK; 130162. 